\title{
Mid-Infrared Strong Field Ionization Angular Distributions ${ }^{1}$
}

\author{
F. Catoire ${ }^{a} *$, C. I. Blaga ${ }^{a}$, E. Sistrunk ${ }^{a}$, H. G. Muller ${ }^{b}$, P. Agostini ${ }^{a}$, and L. F. DiMauro ${ }^{a}$ ** \\ ${ }^{a}$ Department of Physics, The Ohio State University, OH, 43210 Columbus \\ ${ }^{b}$ FOM AMOLF 407 Kruislaan, 1098 SJ Amsterdam, The Netherlands \\ *e-mail: catoire@mps.ohio-state.edu \\ **e-mail:dimauro@mps.ohio-state.edu \\ Received March 22, 2009
}

\begin{abstract}
We present a study of the photoelectrons energy distribution from ionization of Argon by a linearly polarized, intense, mid-infrared laser field with special attention to the recently discovered Low Energy Structure (LES) [1] whose origin is not yet fully understood. In this paper we will go deeper in the analysis of the LES by studying its angular distribution and examine its behavior in circularly polarized light.
\end{abstract}

PACS numbers: 40.

DOI: $10.1134 / \mathrm{S} 1054660 \mathrm{X} 09150079$

\section{INTRODUCTION}

At the beginning of the sixties a revolutionary device, the Q-switched Ruby laser, appeared in a few laboratories around the world. The radiation was intense enough to make observable many new phenomena among which the air breakdown was one of the most intriguing. The first clean measurements of the amount of ionization induced in rare gases were carried out at the Ohio State University [2]. This work, and a few others, inspired Keldysh's famous 1965 paper [3]. The US team, for some reason, dropped the subject after a short publication but Voronov and Delone in Moscow, started systematic studies of multiphoton ionization of atoms [4] soon followed by Mainfray in France and others. Since these pioneering times more than forty years have elapsed after which both multiphoton and tunnel strong field ionization and, particularly, the corresponding photoelectron spectra are usually considered as well understood. The tunneling regime, in which we are interested here, is characterized by a Keldysh parameter $\gamma$ [5] smaller than 1 , easily achieved at long wavelengths in the midinfrared $[1,6]$. In this regime, the photoelectrons can be nicely separated into direct (most of them) and backscattered. This distribution is readily explained by assuming that electrons tunnel out near the peaks of the field, (given the exponential dependence of the tunneling probability on the field strength) and acquire a drift motion whose kinetic energy has an upper bound which can be expressed as a function of the ponderomotive energy $E_{k} \leq 2 U_{p}$ in the short pulse regime. The profile of the spectrum simply reflects the exponential dependence of the tunneling probability that is proportional to $\exp \left(-\frac{2\left(2 I_{p}\right)^{3 / 2}}{3 F}\right)$, where $F$ is the

\footnotetext{
${ }^{1}$ The article is published in the original.
}

field strength and $I_{p}$ the ionization potential. A few electrons recollide with their parent ion and elastically back-rescatter and gain more energy from the field, forming the well documented plateau between $2 U_{p}$ and $10 U_{p}$ [7]. This combination of the tunneling probability and classical mechanics, which is called the semiclassical model, predicts well the envelope of the spectrum [8] but is obviously unable to describe the discrete nature of the energy spectrum, the so-called ATI peaks [9]. Quantum calculations using a Strong Field Approximation (SFA) [10-12] (in the following, the KFR model will refer explicitly to this reference, i.e., an infinitely long pulse and SFA otherwise) or a numerical solution of the time-dependent Schrödinger equation (TDSE) yield a comb of peaks separated by the photon energy, globally similar to the spectra predicted by the semi-classical model. Energy conservation moreover implies a global offset proportional to the field intensity $[13,14]$. If the spectrum, as it is usually the case, is recorded with a focused beam, the intensity distribution inside the focus tends to blur the comb structure especially at wavelengths larger than $1 \mu \mathrm{m}$ due to the combination of the small photon energy, the resolution of the apparatus and the high intensity.

The general structure of the spectra, in the tunneling regime, is therefore well understood and, since the longer the wavelength (at constant intensity) the lower $\gamma$ and the deeper into that regime, no deviation from the model we just outlined is expected. At variance with this expectation, a low energy spike-like structure which had eluded observation hitherto, was discovered first in the xenon and argon spectra at $3.6 \mu \mathrm{m}$, then in essentially all the investigated atomic and molecular targets for wavelengths between 1.0 and $3.6 \mu \mathrm{m}$ as long as $\gamma \leq 0.7$. Although not much attention had really been given to that part of the spectra until these investigations at mid-infrared wavelengths, this prominent 


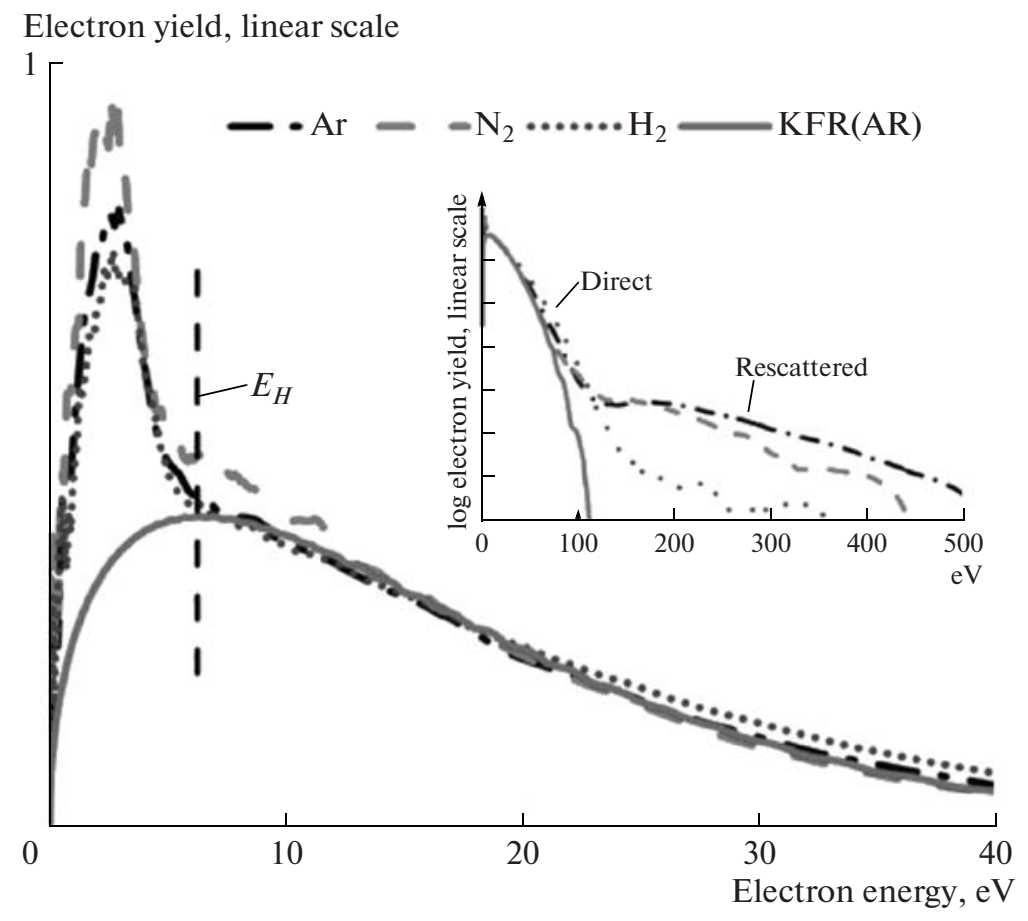

Fig. 1. Energy distribution at $2 \mu \mathrm{m}$ and a peak intensity of $1.3 \times 10^{14} \mathrm{~W} / \mathrm{cm}^{2}$ for $\mathrm{Ar}$ (dot-dash curve), $\mathrm{N}_{2}$ (dash curve), $\mathrm{H}_{2}$ (dot curve) and KFR simulation (full curve) performed for Ar.

feature came as a surprise mostly because of the discrepancy with strong field approximation calculations. Indeed, the KFR model clearly misses the structure while numerical solutions of the TDSE, when possible, do reproduce it. A typical spectrum compared to the KFR strong field approximation calculation clearly illustrates the disagreement in Fig. 1. The two spectra are normalized to the tail of distribution. With this normalization, the experimental spectrum obviously towers above the theoretical one in an energy span of a few volts. For the sake of quantification, the width of the structure can be (arbitrarily) defined by the energy where a break in the slope is observed (the $E_{H}$ broken line as indicated in Fig. 1). In a previous paper we have reported some of the observations regarding the LES and in particular the remarkable scaling properties of its width [1] without, however, reaching an understanding comparable to that of the rest of the spectrum. All these observations were carried out in linearly polarized light and a detection direction along the polarization. In this paper, we complete them by a study of, first, the angular distributions and second, the behavior of the spike in circularly polarized light.

\section{DESCRIPTION OF THE APPARATUS}

The apparatus has been described in some details in $[1,6]$. Briefly, the $2 \mu \mathrm{m}$ source is a modified commercial traveling-wave optical parametric amplifier (Light Conversion, Model HE-TOPAS). The OPA is pumped by $4 \mathrm{~mJ}, 50$ fs pulses originating from a $1 \mathrm{kHz}$ titanium sapphire amplifier system operating at $0.79 \mu \mathrm{m}$. First, superfluorescence is generated in $\beta$ barium borate (BBO) crystal pumped by a small fraction of the titanium sapphire light. In subsequent passes, the same crystal acts as a preamplifier of the wavelength selected signal beam (in this case $1.3 \mu \mathrm{m}$ ). Finally, a $0.5 \mathrm{~mm}$ long BBO crystal acts as a difference frequency generator, and is synchronously seeded by the amplified signal and the remaining pump beam in a non-collinear geometry that allows to spatially separate the pump, the signal and the idler beams. The pulse duration of $50 \mathrm{fs}(7-8$ cycles at $2 \mu \mathrm{m})$ was measured using interferometric autocorrelation. The peak intensity can be evaluated either from the focal waist, directly characterized with a thermal camera, and the estimate based on the $2 U_{p}$ break in the photoelectron spectrum (correlated to the $10 U_{p}$ break when possible, i.e., the tunneling regime). The two methods agree within $20 \%$. The photoelectron energy distributions are recorded using a linear time-of-flight spectrometer. The laser pulses are focused by an effective $f=10$ lens and the resulting electrons are detected at the end of a field-free, magnetically shielded flight tube $(23 \mathrm{~cm})$ by a $40 \mathrm{~mm}$ diameter micro-channel plate detector in a chevron configuration. The angular distributions were recorded with a $1.5^{\circ} \mathrm{FWHM}$ angular resolution using a $6 \mathrm{~mm}$ diameter pinhole to restrict the collection angle. The light polarization was rotated using a half waveplate. For the circularly polarized light a quarter-wave plate was inserted just before the 


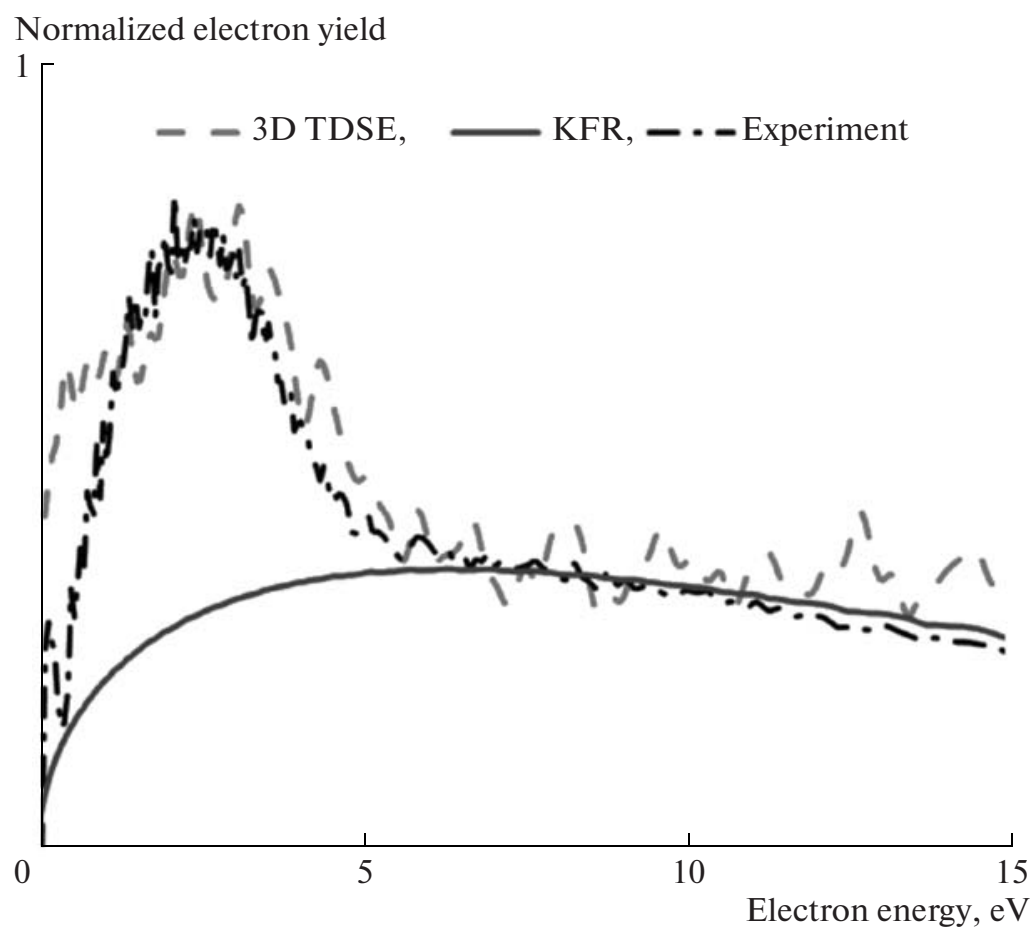

Fig. 2. Comparison of TDSE $3 D$ (dashed curve), KFR (full curve) and the experiment (dot-dash curve) for Ar at $2 \mu \mathrm{m}$ and a peak intensity of $1.3 \times 10^{14} \mathrm{~W} / \mathrm{cm}^{2}$.

focusing lens. The MCP signal is amplified and recorded by a time-to-digital converter with a 1 ns resolution. Depending on the intensity, the gas density is adjusted to produce roughly 1 electron per laser shot, minimizing space charge effects.

\section{THEORETICAL MODELS}

Both the SFA theory using the length gauge [15] and the numerical solution of the TDSE in the single active electron approximation have been used to analyze the data [16]. In the latter, the ground state of argon is calculated numerically by using a static potential (which includes the proper excited states [17]) and is then propagated on a spatio-temporal grid. The energy and angle resolved analysis of the wave function is performed by using the window technique based on the resolvent operator. The velocity gauge has been used for reasons discussed in [18]. In the case of the circular polarization we have used the KFR model [13, $19,20]$.

\section{RESULTS AND DISCUSSIONS}

Before discussing the results, we recall our analysis and conclusions about the LES. At variance with SFA, the TDSE calculations for argon, integrated over the focal volume, reasonably reproduce the experimental data (Fig. 2). We therefore assume that whatever is causing the LES, this ingredient is absent from SFA. In this respect, SFA is a first order calculation and therefore does not take the interaction of the recolliding electron with the ion core into account. Besides, the use a Volkov state which neglects the effect of the Coulomb potential in the final state is another well known deficiency of this approach. Finally, the possible effect of excited states is not included in the calculation. On the other hand, in the TDSE calculation everything is virtually exact as long as the single active electron approximation is valid. We have worked backwards, trying to modify the TDSE parameters (both atomic and laser) in order to suppress the LES. Thus, the contribution of the excited states can be controlled by using a potential which supports only one bound state, by suppressing the Coulomb long range tail. Since the resulting distribution does not globally change, it can be concluded that the excited states and the Coulomb tail are not directly responsible for the LES. This is confirmed by a Coulomb-corrected SFA calculation which does not significantly influence the distribution of Fig. 1. This designates the rescattering as a likely origin of the LES. Furthermore, reducing the pulse to a single cycle, TDSE calculations remain globally unchanged, suggesting that the LES is an intra-cycle effect.

In conclusion, the re-collision process seems to play a role in building the LES although no definite process that would yield an excess of electrons with respect to the standard SFA can be identified at this point. To further investigate this phenomenon, angular distributions with linear polarization as well as 


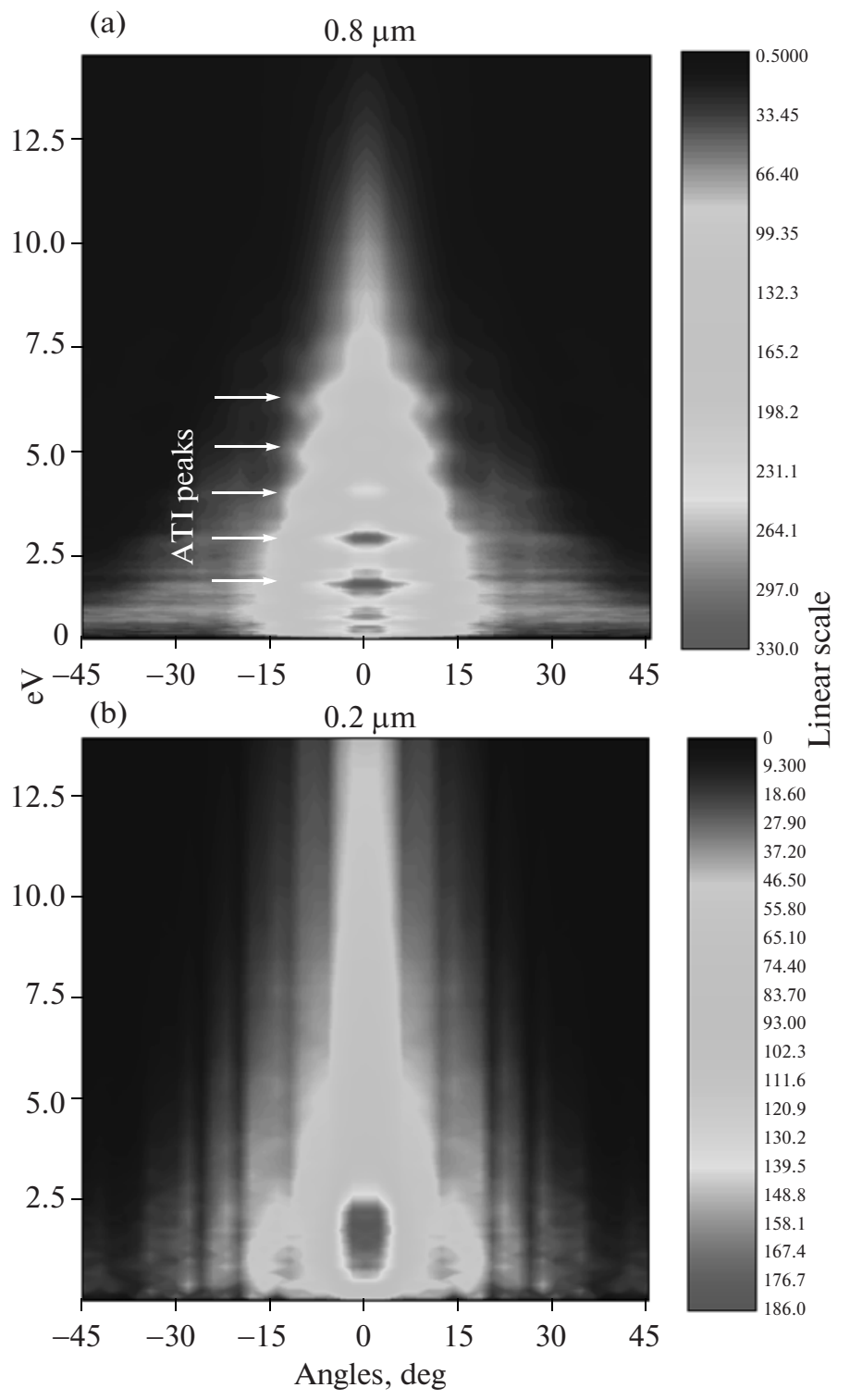

Fig. 3. (a) Probability of ionization as a function of ejection angle and energy at $0.8 \mu \mathrm{m}$, a peak intensity of $1 \times 10^{14} \mathrm{~W} / \mathrm{cm}^{2}\left(U_{p}=\right.$ $6 \mathrm{eV}$ and $\gamma=1.15)$. (b) Same as Fig. 3a but for at $2 \mu \mathrm{m}$ and a peak intensity of $1 \times 10^{14} \mathrm{~W} / \mathrm{cm}^{2}\left(U_{p}=37 \mathrm{eV}\right.$ and $\left.\gamma=0.46\right)$.

spectra with circular polarization have been recorded in the tunneling regime for argon.

\subsection{Angular Distributions with Linear Polarization}

Figures $3 \mathrm{a}$ and $3 \mathrm{~b}$ show the experimental electron count as a function of the kinetic energy and the angle between the polarization and the detection direction for wavelengths equal to 0.8 (Fig. 3a) and $2.0 \mu \mathrm{m}$ (Fig. 3b), at the same peak intensity $I=1 \times 10^{14} \mathrm{~W} / \mathrm{cm}^{2}$. The ATI structure (peaks spaced by the photon energy) dominates in Fig. 3a while in Fig. $3 b$ this structure is blurred. In Fig. $3 b$ the color coding makes the LES apparent (red). The wavelength scaling of the LES (energy range from 2.25 to $2.75 \mathrm{eV}$ ) angular distribution full width half maximum (FWHM) of the distri- butions at 0.8 and $2.0 \mu \mathrm{m}$ is obtained from the plots in Fig. 4.

The two area-normalized curves exhibit the same Gaussian-like shape centered along the laser polarization $\left(\theta=0^{\circ}\right)$ with $30^{\circ}$ and $10^{\circ} \mathrm{FWHM}$ respectively, about three times narrower at the longer wavelength. For comparison, KFR calculations plotted in this graph show qualitatively a narrowing of central peak FWHM but a poor quantitative agreement of both the FWHM and the position of the peaks. This question will be discussed in detail elsewhere.

The narrowing of the angular distribution could be qualitatively reminiscent of some effects of the Coulomb focusing [21]. In [21], the experimental electron momentum distributions (supported by classical simulations) show that the distribution narrows when the 


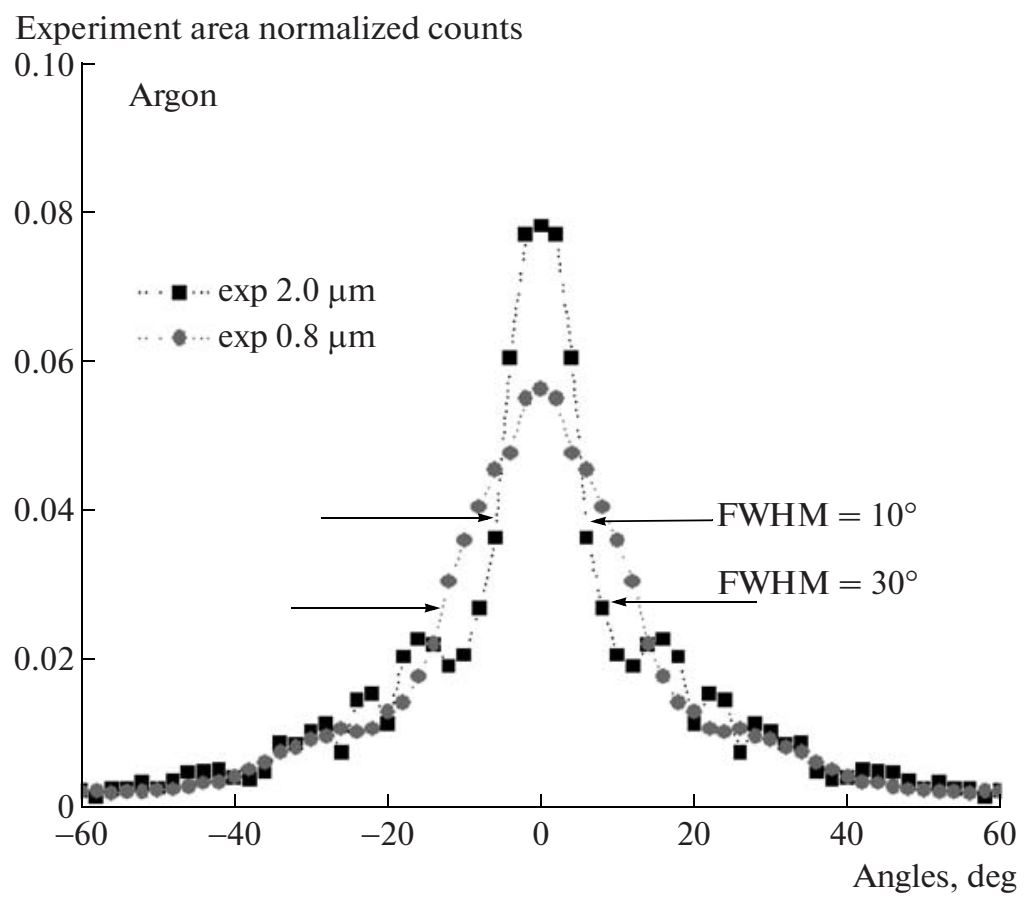

Fig. 4. Plot the photoelectron spectrum as a function of for the same condition as Fig. 3a (circles) and Fig. 3b (squares) but integrated in energy from 2.25 up to $2.75 \mathrm{eV}$. The results of the KFR simulation is shown for $2.0 \mu \mathrm{m}$ (full curve) and for $0.8 \mu \mathrm{m}$ (dashed curve).

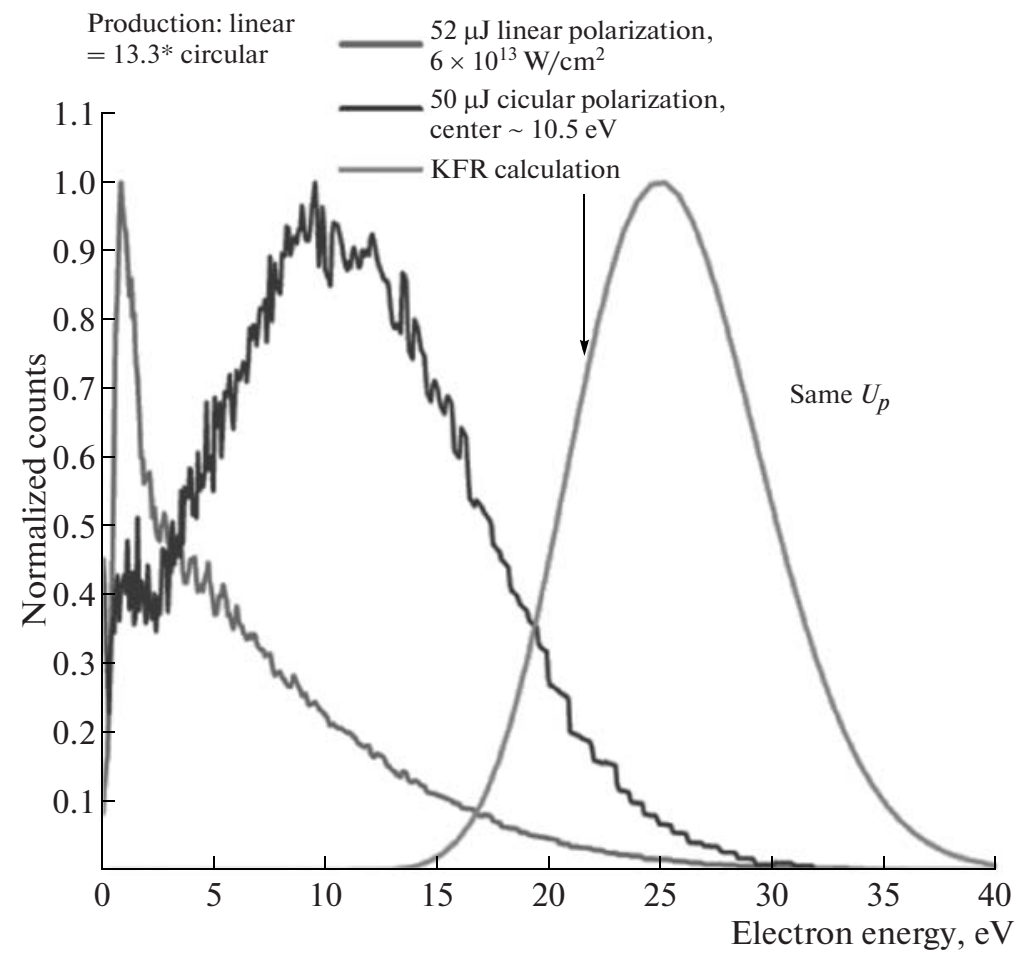

Fig. 5. Photoelectron spectrum in Argon at $6 \times 10^{13} \mathrm{~W} / \mathrm{cm}^{2}$ at a wavelength of $2 \mu \mathrm{m}$ in the linear polarization (light grey curve) and circularly polarized light (dark grey curve). The smooth curve represents the KFR simulation in the same laser conditions. The arrow indicates the value of $U_{p}$. 


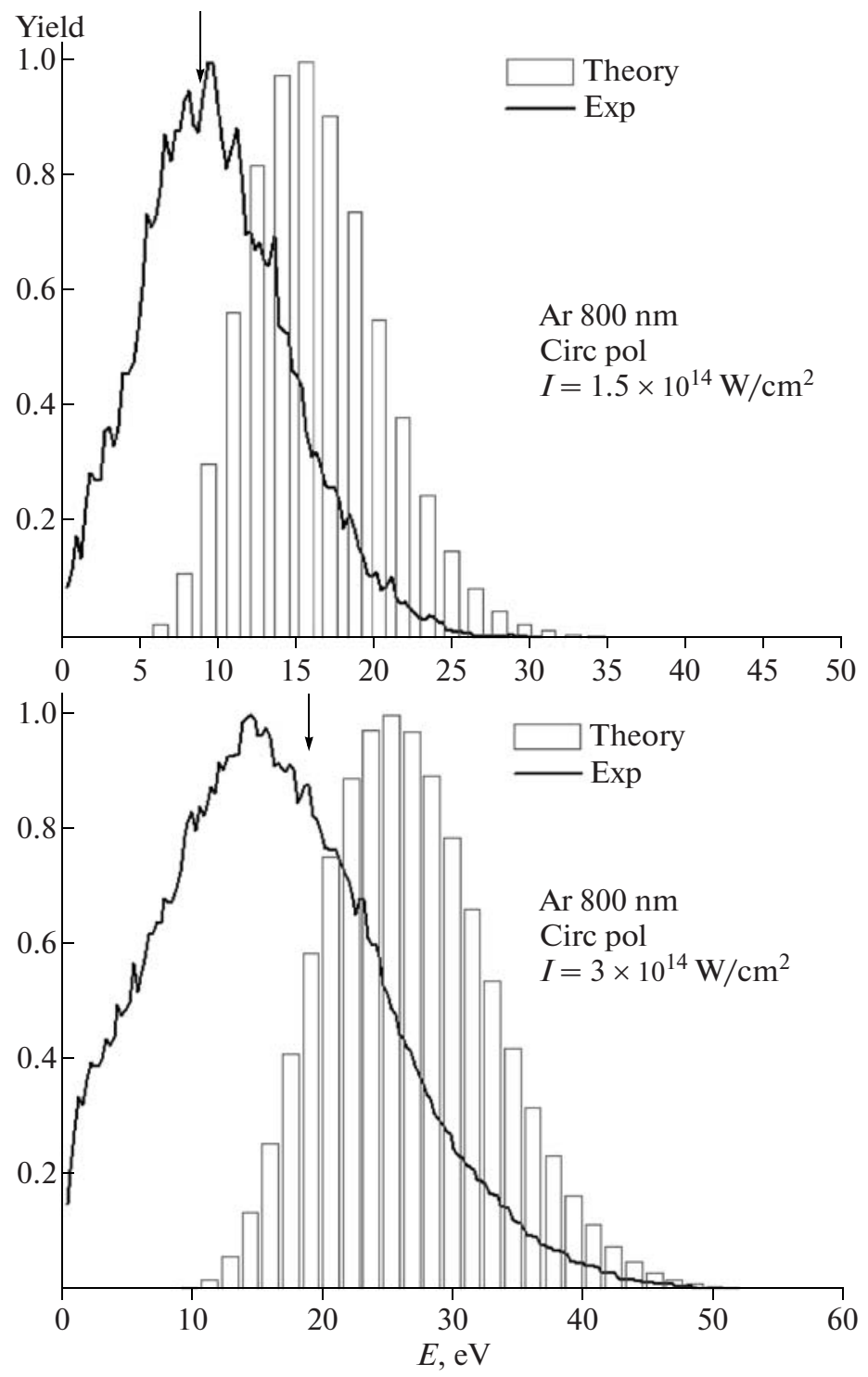

Fig. 6. (a) Photoelectron spectrum at $0.8 \mu \mathrm{m}$ and peak intensity $1.5 \times 10^{14} \mathrm{~W} / \mathrm{cm}^{2}$. The full curve is the experiment and the bar curve is the KFR calculation. The arrow indicates the value of $U_{p}$. (b) Photoelectron spectrum at $0.8 \mu \mathrm{m}$ for a peak intensity of 3 $\times 10^{14} \mathrm{~W} / \mathrm{cm}^{2}$. The legend is the same as Fig. $6 \mathrm{~b}$.

wavelength gets shorter. This phenomenon is explained by the fact that at short wavelengths, the spread of the returning electrons as well as the returning kinetic energy are smaller. Hence the interaction with the core is expected to be stronger at short wavelengths, resulting in a narrowing of the momentum distribution. At variance with these observations, the observed narrowing of the LES occurs at longer wavelength and therefore cannot be assigned to the Coulomb focusing.

Besides the LES angular distributions, clear (albeit weaker) sideband structures are visible in Fig. 3b. These sidebands appear at angles of about $17.0^{\circ} \pm$ $1.5^{\circ}, 22.5^{\circ} \pm 1.5^{\circ}$, and $27^{\circ} \pm 1.5^{\circ}$ that will be discussed elsewhere and can, somehow, be interpreted within the frame of the first order of the Dyson equation showing a different origin than the LES.

In order to further clarify the contribution of recollision processes, we have measured the photoelectron distribution in the case of circularly polarized light where recollisions are strongly suppressed.

\subsection{Photoelectron Spectra in Circular Polarization}

In [1] we have reported that the LES is strongly suppressed in circularly polarized light. This is shown again in Fig. 5. Clearly the LES structure in the range $0-2.5 \mathrm{eV}$ in linearly polarized light essentially vanishes in the circularly polarized case, at the same average intensity. Figure 5 moreover reveals the whole spec- 
trum as well as the KFR spectrum calculated for a single intensity equal to the experimental peak intensity. The classical analysis of the kinematics of an electron in an electric field is well known and will not be reproduced here. In linear polarization the expression of the kinetic energy as a function of the initial phase $\phi$ is $E_{k}^{L}=2 U_{p} \sin ^{2}(\phi)+U_{p}$ having a maximum of $3 U_{p}$ which is reduced to $2 U_{p}$ in a short pulse. In circular polarization the average kinetic energy $E_{k}^{C}=2 U_{p}$ from which $U_{p}$ has to be subtracted for a short pulse. The latter quantity does not depend upon the birth moment and is then equal to $U_{p}$. The photoelectron spectrum of a circularly polarized light is then expected to be centered around $U_{p}$ and the width of the spectrum depends of the pulse duration. KFR theory for circular polarization [13] with $U_{p}=22.4 \mathrm{eV}$ predicts a peak for a kinetic energy of $27 \mathrm{eV}$ for a short pulse. The experimental photoelectron spectrum exhibits a bell-shaped spectrum with a maximum centered around $12 \mathrm{eV}$, i.e., significantly lower than the KFR or the classical mechanics predictions. However it should be stressed that no attempt has been made to model the intensity distribution of the focused beam. It is clear that the averaging should lower the peak value and improve the comparison with the experiment. At $0.8 \mu \mathrm{m}$ a smaller discrepancy is found (Figs. 6a and $6 \mathrm{~b}$ for two intensities) with probably the same explanation. Regarding the behavior at low energy, in the range of the LES, it appears that KFR is in better agreement with the experiment for circular polarization. In linear polarization, KFR reasonably describes the tail of the spectrum (up to $2 U_{p}$ ) but fails to reproduce the LES. The ensemble of the observations leads to the conclusion that the LES is somehow linked to the rescattering.

\section{CONCLUSIONS}

In summary, after reviewing the main characteristics of the LES, we have presented the photoelectron angular distributions for energy range including the LES. The narrowing of the angular distribution at $2 \mu \mathrm{m}$ suggests the influence of re-collision and/or Coulomb effect. The wavelength dependence of the Coulomb focusing does not agree with the one of the LES then eliminating this process as giving birth to the LES. In circularly polarized light, the LES is essentially suppressed which most likely indicates that the LES is linked to rescattering processes.

\section{ACKNOWLEDGMENTS}

This work was performed with support from the National Science Foundation under contract PHY0653022. L.F.D. acknowledges support from the Hagenlocker chair.

\section{REFERENCES}

1. C. I. Blaga, F. Catoire, G. G. Paulus, H. G. Muller, P. Agostini, and L. F. DiMauro, Nature Phys. (2009, in press).

2. R. G. Tomlinson, Phys. Rev. Lett. 14, 489 (1965).

3. L. V. Keldysh, Sov. Phys. JETP 20, 1018 (1965).

4. G. S. Voronov and N. B. Delone, Sov. Phys. JETP 23, 54 (1966).

5. L. V. Keldysh, Sov. Phys. JETP 20, 1945 (1964).

6. P. Colosimo, G. Doumy, C. I. Blaga, J. Wheeler, C. Hauri, J. Tate, F. Catoire, R. Chirla, A. M. March, G. G. Paulus, H. G. Muller, P. Agostini, and L. F. DiMauro, Nature Phys. 4, 386 (2008).

7. G. G. Paulus, W. Nicklich, Huale Xu, P. Lambropoulos, and H. Walther, Phys. Rev. Lett. 72, 2851 (1994).

8. B. Sheehy, R. Lafon, M. Widmer, B. Walker, L. F. DiMauro, P. A. Agostini, and K. C. Kulander, Phys. Rev. A 58, 3942 (1998).

9. P. Agostini, F. Fabre, G. Mainfray, G. Petite, and N. K. Rahman, Phys. Rev. Lett. 42, 1127 (1979).

10. H. R. Reiss, Phys. Rev. A 22, 1786 (1980).

11. L. V. Keldysh, Zh. Eksp. Teor. Fiz. 47, 1945 (1964) [Sov. Phys. JETP 20, 1307 (1965)].

12. F. Faisal, J. Phys. B 6, L312 (1973).

13. H. R. Reiss, Phys. Rev. A 22, 1786 (1980).

14. J. Tate, J. Phys. Rev. Lett. 98, 013901 (2007).

15. D. B. Miloševic, G. G. Paulus, D. Bauer, and W. Becker, J. Phys. B: At. Mol. Opt. Phys. 39, R203 (2006).

16. H. G. Muller and F. C. Kooiman, Phys. Rev. Lett. 81, 1207 (1998).

17. C. E. Moore, Atomic Energy Levels (NBS, Washington, 1949), Vol. I, p. 212.

18. H. G. Muller, Laser Phys. 9, 138 (1999).

19. P. B. Corkum, N. H. Burnett, and F. Brunel, Phys. Rev. Lett. 62, 1259 (1989).

20. P. H. Bucksbaum, M. Bashkansky, and D. W. Schumacher, Phys. Rev. A 37, 3615 (1988).

21. D. Comtois, D. Zeidler1, H. Pépin, J. C. Kieffer, D. M. Villeneuve, and P. B. Corkum, J. Phys. B 38, 1923 (2005). 